\title{
ON COMMUTATIVITY OF ENDOMORPHISM RINGS OF IDEALS
}

\author{
S. ALAMELU
}

\begin{abstract}
ABSTRACr. Let $R$ be a commutative noetherian ring with $\operatorname{Hom}_{R}(I, I)$ commutative for all ideals $I$. Then the total quotient ring of $R$ is quasi-Frobenius. This settles a conjecture of W. V. Vasconcelos [2].
\end{abstract}

All rings considered are commutative with identity. It is well known that if $R$ is an integral domain, $\operatorname{Hom}_{R}(I, I)$ is always commutative for every ideal $I$ of $R$. The main purpose of this paper is to prove the following theorem. ${ }^{1}$

THEOREM. Let $R$ be a commutative ring whose total quotient ring $K$ is noetherian. Then $\operatorname{Hom}_{R}(I, I)$ is commutative for every ideal $I$ of $R$ if and only if $K$ is quasi-Frobenius.

The theorem is a consequence of two lemmas.

LEMMA 1. Let $R$ be a noetherian ring which is its own total quotient ring. If $\operatorname{Hom}_{R}(I, I)$ is commutative for every ideal I of $R$, then (0) has no embedded prime factors.

Proof. Suppose (0) has embedded prime factors. Let $(0)=Q_{1} \cap \cdots \cap$ $Q_{r} \cap Q_{r+1} \cap \cdots \cap Q_{n}$, where the $Q_{i}$ are $P_{i}$-primary. We can assume $P_{i}$, $1 \leqq i \leqq r$, are the isolated primes of $R$, and $P_{j}, r+1 \leqq j \leqq n$, are the embedded primes. There exists an element $y \in Q_{r+1} \cap \cdots \cap Q_{n}$ such that $y \notin P_{1} \cup \cdots \cup P_{r}$. Let $x$ be an element in $Q_{1} \cap \cdots \cap Q_{r}$. Let $I$ be the ideal generated by $x$ and $y$. We shall prove that $\operatorname{Hom}_{R}(I, I)$ is not commutative. Consider the map $F: I \rightarrow I$ given by $x \rightarrow x, y \rightarrow 0$, and in general $a x+b y \rightarrow a x$. Since $(x) \cap(y)=(0), a x+b y=0$ implies $a x=0$ and therefore this is a welldefined map. It is clearly a homomorphism. Next, consider the map $G: I \rightarrow I$ given by $x \rightarrow 0, y \rightarrow t x$, and $a x+b y \rightarrow b t x$ (where $t$ is to be chosen as follows). Since $y \notin P_{1} \cup \cdots \cup P_{r}$, Ann $y \subset P_{1} \cap \cdots \cap P_{r}$. Therefore

Received by the editors April 3, 1972.

AMS (MOS) subject classifications (1969). Primary 1320; Secondary 1325.

Key words and phrases. Endomorphism ring, isolated prime ideal, embedded prime ideal, total quotient ring, quasi-Frobenius ring, unmixed ideal.

${ }^{1}$ I learn from the referee that our theorem has also been proved independently by $S$. Cox, Commutative endomorphism rings to appear in Pacific J. Math.

(c) American Mathematical Society 1973 
Ann $y$ is nilpotent, say (Ann $y)^{n}=(0)$. Let $m$ be the smallest integer so that $(\text { Ann } y)^{m} x=0$. Let $t$ be an element of (Ann $\left.y\right)^{m-1}$ such that $t x \neq 0$. Then $s t x=0 \forall s \in$ Ann $y$ (if $m-1=0$, then $t=1$ ). The map is well defined, for suppose $a x+b y=0$, i.e. $a x=0$, and $b y=0$. Then $b \in$ Ann $y$ implies $b t x=0$. And $G$ is a homomorphism. $F \circ G(y)=t x$, and $G \circ F(y)=0$ contradicting our assumption that $\operatorname{Hom}_{R}(I, I)$ is commutative.

LemMa 2. Let $R$ be an Artinian local ring with the unique prime ideal $P$. Suppose $\operatorname{Hom}_{R}(I, I)$ is commutative for every ideal $I$, then $(0)$ is irreducible.

Proof. Suppose (0) is not irreducible. Let $(0)=Q_{1} \cap Q_{2}$ such that $Q_{1} \neq(0), Q_{2} \neq(0)$. Take $0 \neq x \in Q_{1}, 0 \neq y \in Q_{2}$. Let $I$ be the ideal generated by $x$ and $y$. Consider the map $F: I \rightarrow I$ given by $x \rightarrow x, y \rightarrow 0$ and extend by linearity. This is a well-defined homomorphism since $(x) \cap(y)=(0)$. Let $G:(I) \rightarrow I$ be given by $x \rightarrow 0, y \rightarrow \alpha x$, and extend by linearity. $\alpha$ is chosen such that $s^{\prime} \alpha x=0, s^{\prime} \in$ Ann $y$. Since Ann $y$ is finitely generated and nilpotent, we can always find such an $\alpha$. Clearly $F G(y) \neq G F(y)$ contradiction.

We now proceed to the proof of the theorem. First we make a few remarks of a general nature.

REMARK 1. Let $M$ be any module, $N$ a torsion-free $R$-module (no nonzero element of $N$ can be annihilated by a nonzero divisor) and $S$ a multiplicative set of nonzero divisors of $R$. Then $\left(\operatorname{Hom}_{R}(M, N)\right)_{S} \rightarrow$ $\operatorname{Hom}_{R_{S}}\left(M_{S}, N_{S}\right)$ is a monomorphism. The map is given by $f / s \rightarrow f_{S}^{*}$, $f_{S}^{*}\left(m / s^{\prime}\right)=f(x) / s s^{\prime}$. If $M$ is finitely generated then the map is a monomorphism for any multiplicative closed set of nonzero elements, and for any $N$.

REMARK 2. Let $I$ be a finitely generated ideal of $R$. Then $\left(\operatorname{Hom}_{R}(I, I)\right)_{S} \rightarrow$ $\operatorname{Hom}_{R_{S}}\left(I_{S}, I_{S}\right)$ is an isomorphism of rings, where $S$ is a multiplicative closed set of nonzero divisors. If $I$ is finitely related then $S$ can be allowed to be any multiplicative set of nonzero elements.

Let $0 \rightarrow K \rightarrow F \rightarrow I \rightarrow 0$ be an exact sequence where $F$ is a finitely generated free module. Then we have the commutative diagram

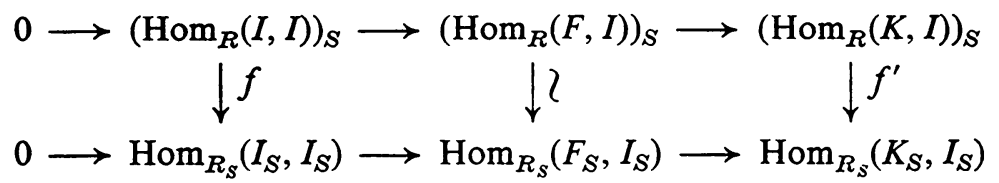

The middle map is an isomorphism and $f$ and $f^{\prime}$ are monomorphisms. This shows that $f$ is an epimorphism and hence an isomorphism. It is clear that $f$ is an isomorphism of rings.

Let $K$ denote the total quotient ring of $R$ and $S$ the set of nonzero divisors of $R$. Let us suppose that $K$ is quasi-Frobenius. Then for any ideal $J$ of $K$, any $K$-homomorphisms $J \rightarrow J$ is given by multiplication by an 
element of $K$ and hence $\operatorname{Hom}_{K}(J, J)$ is commutative. By Remark 1, for any ideal $I$ of $R$, the map $\left(\operatorname{Hom}_{R}(I, I)\right)_{S} \rightarrow \operatorname{Hom}_{K}\left(I_{S}, I_{S}\right)$ is an injection. Therefore $\operatorname{Hom}_{R}(I, I)$ is commutative.

Next suppose $\operatorname{Hom}_{R}(I, I)$ is commutative for all ideals $I$ and $K$ is noetherian. If $J$ is any ideal of $K$, there exists a finitely generated ideal $I$ of $R$ such that $I_{S}=J$. By Remark $2,\left(\operatorname{Hom}_{R}(I, I)\right)_{S} \cong \operatorname{Hom}_{K}(J, J)$. This shows that $\operatorname{Hom}_{K}(J, J)$ is commutative for all ideals $J$ of $K$. Thus by Lemma $1,(0)$ is unmixed in $K$. Since every ideal of $K$ is finitely related, $\left(\operatorname{Hom}_{K}(J, J)\right)_{P} \rightarrow \operatorname{Hom}_{K_{P}}\left(J_{P}, J_{P}\right)$ is an isomorphism for any prime ideal $P$ of $K$. By Lemma 2, (0) is irreducible in $K_{P}$ and hence the primary components of (0) in $K$ are irreducible.

We now recall the following theorem of $\mathrm{H}$. Bass [1].

Let $R$ be a noetherian ring and $K$ its total quotient ring. The following conditions are equivalent.

(1) $K$ is $K$-injective,

(2) the zero ideal of $R$ is unmixed and all of its primary components are irreducible.

In the present case, by taking $K=R$, the theorem follows.

The author wishes to express her best thanks to Dr. K. R. Nagarajan for all his encouragement and guidance.

\section{REFERENCES}

1. H. Bass, On the ubiquity of Gorenstein rings, Math. Z. 83 (1963), 8-28. MR 27 \#3669.

2. W. V. Vasconcelos, On commutative endomorphism rings, Pacific J. Math. 35 (1970), 795-798. MR 43 \#4812.

Department of Mathematics, Madurai University, Madurai-2, Tamilnadu, INDIA 